\title{
Reliability Analysis for Degradation of Locomotive Wheels using Parametric Bayesian Approach
}

\author{
Jing Lin, ${ }^{a * \dagger}$ Matthias Asplund ${ }^{\mathrm{a}, \mathrm{b}}$ and Aditya Parida ${ }^{\mathrm{a}}$
}

This paper undertakes a reliability study using a Bayesian survival analysis framework to explore the impact of a locomotive wheel's installed position on its service lifetime and to predict its reliability characteristics. The Bayesian Exponential Regression Model, Bayesian Weibull Regression Model and Bayesian Log-normal Regression Model are used to analyze the lifetime of locomotive wheels using degradation data and taking into account the position of the wheel. This position is described by three different discrete covariates: the bogie, the axle and the side of the locomotive where the wheel is mounted. The goal is to determine reliability, failure distribution and optimal maintenance strategies for the wheel. The results show that: (i) under specified assumptions and a given topography, the position of the locomotive wheel could influence its reliability and lifetime; (ii) the Bayesian Log-normal Regression Model is a useful tool. Copyright ๑ 2013 John Wiley \& Sons, Ltd.

Keywords: reliability; Bayesian survival analysis; locomotive wheels; Deviance Information Criterion (DIC); Markov Chain Monte Carlo (MCMC)

\section{Introduction}

$\mathrm{T}$ he service life of a railroad wheel can be significantly reduced due to failure or damage, leading to excessive cost and accelerated deterioration. Damage data show that a major proportion of wheel damage stems from degradation.

In order to monitor the performance of wheels and make replacements before adverse effects occur, the railway industry uses both preventive and predictive maintenance. By predicting the wear of train wheels (Johansson \& Andersson ${ }^{1}$; Braghin et al. ${ }^{2}$; Tassini et al. ${ }^{3}$ ), fatigue (Bernasconi et al. ${ }^{4}$; Liu, et al. ${ }^{5}$ ), tribological aspects $\left(\right.$ Clayton $^{6}$ ) and failures (Yang \& Letourneau ${ }^{7}$ ), the railway industry can design strategies for different types of preventative maintenance (re-profiled, lubrication, etc.) for various time periods (days, months, seasons, running distance, etc.). Software dedicated to predicting wear rate has also been studied recently (Pombo et al. ${ }^{8}$ ). In addition, condition monitoring data have been studied to increase the wheels' lifetime (Skarlatos D, Karakasis K \& Trochidis A ${ }^{9}$; Donato P et al. ${ }^{10}$; Stratman et al. ${ }^{11} ;$ Palo ${ }^{12,13}$ ). A large number of related studies examining both experimental and numerical aspects have been published in the last decade (see above references).

In one common preventive maintenance policy in the Swedish railway company studied, a wheel's diameter is measured after running a certain distance. If it is reduced to a pre-specified height, the wheel is replaced. Otherwise, it is re-profiled or other maintenance strategies are adopted. To optimize maintenance strategies for railway wheels, some researchers have used degradation data to determine reliability and failure distribution (Freitas et al. ${ }^{14,15}$; and the reference therein). However, these studies cannot solve the combined problem of small data samples and incomplete data sets while simultaneously considering the influence of several covariates. For example, to avoid the potential influence of the different locations of wheels, the researchers only consider those on the left side of axle number 1 and on certain specified cars.

Other researchers have noted that the wheel's position on the locomotive could influence degradation. For example, researchers from Canada (Yang \& Letourneau ${ }^{7}$ ) suggest that certain attributes, including a wheel's installed position (right or left), might influence its wear rate, but they do not provide case studies. Freitas and colleagues ${ }^{14}$ point out that 'the degradation of a given wheel might be associated with its position on a given car'; Palo ${ }^{12,13}$ conclude that 'different wheel positions in a bogie show significantly different force signatures'. In a recent seminar in Sweden (Kiruna, April 2012), experts from Norway illustrated their new findings that in a given topography, the wheels installed on the right and the left sides experience different force. Unfortunately, they only illustrated the results with signal charts derived from condition monitoring tools. Nor did they consider the influence of wheel position on degradation.

\footnotetext{
${ }^{a}$ Division of Operation and Maintenance Engineering, Luleå University of Technology, 97187, Luleå, Sweden

${ }^{b}$ Swedish Transport Administration97102, Luleå, Sweden

*Correspondence to: Jing Lin, Division of Operation and Maintenance Engineering, Luleå University of Technology, 97187, Luleå, Sweden.

${ }^{\dagger}$ E-mail: janet.lin@ltu.se
} 
To address the above issues, this paper undertakes a reliability study using a Bayesian survival analysis framework (Ibrahim ${ }^{16}$; Congdon ${ }^{17,18}$; Lin $^{19}$ ) to explore the impact of the wheel's installed position on its service lifetime and to predict its reliability characteristics. The Bayesian Exponential Regression Model, Bayesian Weibull Regression Model and Bayesian Log-normal Regression Model are used to analyze the lifetime of locomotive wheels using degradation data and taking into account the position of the wheel. This position is described by three different discrete covariates: the bogie, the axle and the side of the locomotive where the wheel is mounted. In particular, by introducing the covariate $\mathbf{x}_{i}^{\prime}$ s linear function $\mathbf{x}_{i}^{\prime} \boldsymbol{\beta}$, these three parameter models are constructed depending on the failure rate $\lambda_{i}$ in the exponential model, the log of the rate parameter $\ln \left(\gamma_{i}\right)$ in the Weibull model and the logarithmic mean $\mu_{i}$ in the log-normal models. The contribution of this work is to propose Bayesian survival models, which can solve the combined problem of small data samples and incomplete data sets while simultaneously considering the influence of several covariates. The goal is to determine reliability, failure distribution and optimal maintenance strategies for the wheel.

The organization of this paper is as follows. The introductory section defines the problem. Section 2 describes the data. Section 3 presents three Bayesian survival models. In those models, some parameters depend on the above-mentioned covariates: the bogie, the axle and the side of the locomotive where the wheel is mounted. Section 4 provides the results for a real data set. This section adopts vague priors and a Markov Chain Monte Carlo (MCMC) computational scheme to obtain the parameters' posterior distributions. Section 5 compares the proposed models with Deviance Information Criterion (DIC), mean time to failure (MTTF) predictions and discusses the effect on the results of setting maintenance inspection levels. Finally, Section 6 offers conclusions and comments. We also note our ongoing study in the JVTC (Järnvägstekniskt Centrum, Sweden) program.

\section{Data description}

This paper focuses on the wheels of the locomotive of a cargo train. While two types of locomotives with the same type of wheels are used in cargo trains, we consider only one.

\subsection{Locomotive wheels' degradation data}

As shown in Figure 1, there are two bogies for each locomotive and three axels for each bogie. The installed position of the wheels on a particular locomotive is specified by a bogie number (I, Il-number of bogies on the locomotive), an axel number (1, 2, 3-number of axels for each bogie) and the side of the wheel on the axle (right or left) where each wheel is mounted.

The diameter of a new locomotive wheel in the studied railway company is $1250 \mathrm{~mm}$. In the company's current maintenance strategy, a wheel's diameter is measured after running a certain distance. If it is reduced to $1150 \mathrm{~mm}$, the wheel is replaced by a new one. Otherwise, it is re-profiled or other maintenance strategies are implemented. A threshold level for failure, denoted as $H_{1}$ in this paper, is defined as $100 \mathrm{~mm}\left(H_{1}=1250 \mathrm{~mm}-1150 \mathrm{~mm}\right)$. The wheel's failure condition is assumed to be reached if the diameter reaches $H_{1}$.

The company's complete database also includes the diameters of all locomotive wheels at a given observation time, the total running distances corresponding to their 'time to be maintained (be re-profiled or replaced)', and the wheels' bill of material data, from which we can determine their positions.

\subsection{Locomotive wheels' lifetime data}

In reliability analyses using degradation data, Freitas et al. ${ }^{14,15}$ set up a threshold level as defined in section 2.1. The researchers used the degradation data as the wheels' lifetime data. The censored lifetime data were defined if the degradation measurements had reached the threshold level when they were observed. However, in our study, the way we obtain the wheels' lifetime data and how we define censored data differ from theirs.

We make the following assumptions. First, the wheel's degradation follows a linear path (assumption 1). Second, all maintenance activities are assumed to be effective (assumption 2). A specified special maintenance inspection level is denoted as $\mathrm{H}_{2}$ and $0 \leq H_{2} \leq H_{1}$. If effective maintenance activities are implemented before the degradation height reaches $H_{2}$, the wheel's degradation speed will be lower; if effective maintenance activities are implemented when the degradation height exceeds $H_{2}$, the degradation speed will remain unchanged.

With respect to assumption (1), Freitas et al. ${ }^{14,15}$ show that the linear degradation path is reasonable by plotting the historical records of wheel degradation. In our studies, we calculate the squares of their correlation coefficient for a linear path, which are all
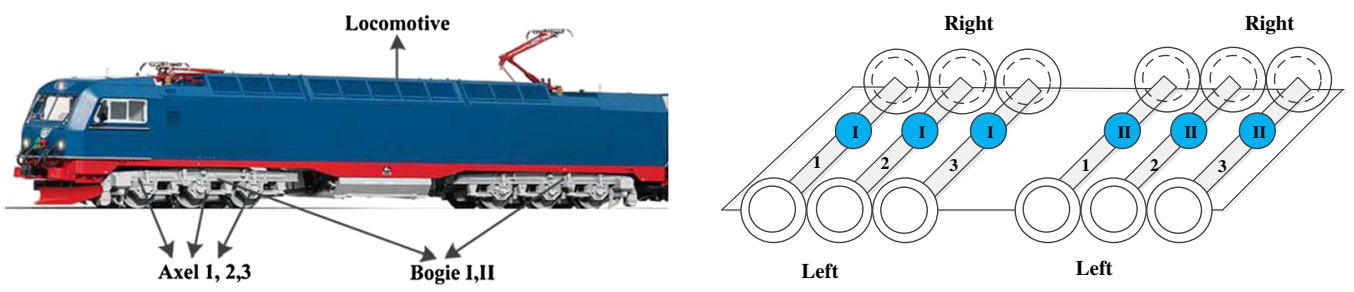

Figure 1. Wheel positions specified in this study 
larger than 0.9 and indicate that the linear degradation path is also a reasonable choice in our case. Assumption (2) is also logical, as the railway company's maintenance activities are intended to prolong the service life of the wheels. However, if maintenance activities are implemented too late (for example, after the degradation height exceeds $\mathrm{H}_{2}$ ), the improvement effects are not significant; at most, they will prevent the degradation from speeding up. In addition, any maintenance activity could affect the wheel's diameter, especially if it is re-profiled.

Based on the above assumptions, and as shown in Figure 2, we take the following steps:

- Step 1: Establish threshold level $H_{1}$. As defined in section 2.1, in Figure 2, we use $H_{1}=100 \mathrm{~mm}$. In addition, according to assumption (2), we establish the specified maintenance inspection level $H_{2}$, where $0 \leq H_{2} \leq H_{1}$. In Figure 2 , we use $H_{2}=50$ mm.

- Step 2: Transfer the diameters of locomotive wheels at observation time $t$ to degradation data; this equals to $1250 \mathrm{~mm}$ minus the corresponding observed diameter. $B_{1}, B_{2}$ and $B_{3}$ are three examples of degradation data shown in Figure 2;

- Step 3: According to assumption (1), we assume a liner degradation path and construct a degradation line using the origin point and the degradation data.

- Step 4: If the degradation data are not less than $H_{1}=100 \mathrm{~mm}$ (for example: $B_{1}$ ), the degradation line will intersect with $H_{1}$. Based on the point of intersection (for example, $E_{1}$ ) and the wheel's failure conditions (see section 2.1), the wheel's lifetime can be determined (for example, $D_{1}$ ).

- Step 5: If the degradation data are less than $H_{1}=100 \mathrm{~mm}$ but more than $H_{2}=50 \mathrm{~mm}$ (for example, $B_{2}$ ), the extended degradation line will also intersect with $H_{1}$ (for example, $E_{2}$ ). Based on the intersection point and according to assumption (2), the wheel's lifetime can be obtained (for example, $D_{2}$ ).

- Step 6: If the degradation data are less than $H_{2}$, which equals to $50 \mathrm{~mm}$ in Figure 2 (for example, $B_{3}$ ), the intersection point and the lifetime can be derived (for example, $E_{3}$ and $D_{3}$ ) as discussed above. However, according to assumption (2), the slope of the extended degradation line could be lower, and intersection point could be changed (for example, $E_{3}^{\prime}$ ). Therefore, the lifetime could be longer than the original prediction (for example, $D_{3}^{\prime}$ ). In this case, the lifetime $D_{3}$ is defined as right censored.

By following the above steps, we can obtain the locomotive wheels' lifetime data and the right-censored data.

We consider the wheels of only one locomotive because for the same locomotive: (i) the wheels' maintenance strategies are similar; (ii) the axle load and running speed can be supposed to have no obvious difference; (iii) the operational environments including routes and climates are expected to be the same. Given these expectations, the installed positions become covariates. Ultimately, we can predict a wheel's lifetime based on its positioning and other important characteristics, including MTTF.

\section{Bayesian survival models}

\subsection{Likelihood construction for right-censored data}

In reliability analysis, the lifetime data are usually incomplete, and only a portion of the individual lifetimes are known. Take the locomotive wheels' lifetime data for example. As discussed in section 2.2, if the degradation data $B_{3}$ is less than the specified maintenance inspection level $H_{2}$, the predicted lifetime $D_{3}$ is viewed as right censored under assumption (2). Therefore, we believe maintenance activities will diminish degradation and the real lifetime $D_{3}^{\prime}$ will exceed the predicted lifetime (see Figure 2).

Right-censored data are often called Type I censoring in the literature; the corresponding likelihood construction problem has been extensively studied (Klein \& Moeschberger ${ }^{20}$; Lawless ${ }^{21}$ ). Suppose there are $n$ individuals whose lifetimes and censoring times are independent. The $i^{\text {th }}$ individual has life time $T_{i}$ and censoring time $L_{i}$. The $T_{i}$ s are assumed to have probability density function (p.d.f.) $f(t)$ and reliability function $R(t)$. The exact lifetime $T_{i}$ of an individual will be observed only if $T_{i} \leq L_{i}$. The lifetime data involving right censoring can be conveniently represented by $n$ pairs of random variables $\left(t_{i}, v_{i}\right)$, where $t_{i}=\min \left(T_{i} L_{i}\right)$ and $v_{i}=1$ if $T_{i} \leq L_{i}$, and $v_{i}=0$ if $T_{i}>L_{i}$. That is, $v_{i}$ indicates whether the lifetime $T_{i}$ is censored or not. The likelihood function is deduced (Klein \& Moeschberger ${ }^{20}$; Lawless ${ }^{21}$ ) as

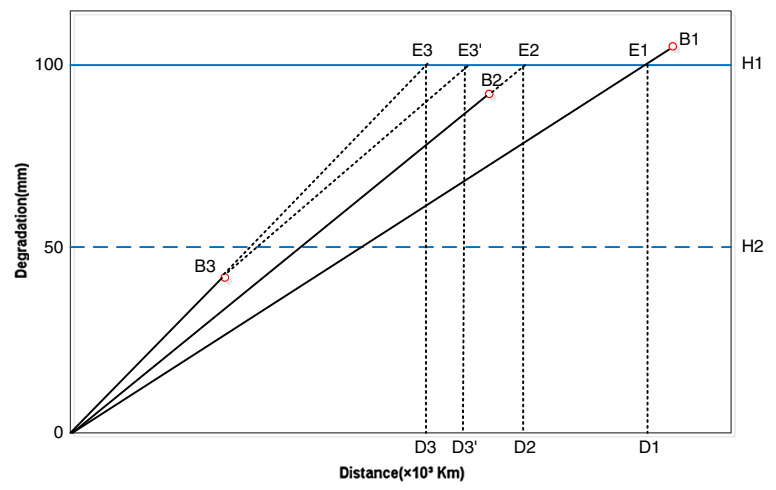

Figure 2. Plot of the wheel degradation data: one example 


$$
L(t)=\prod_{i=1}^{n}\left[f\left(t_{i}\right)\right]^{v_{i}} R\left(t_{i}\right)^{1-v_{i}}
$$

\subsection{Bayesian Exponential Regression Model}

Suppose the lifetimes $\mathbf{t}=\left(t_{1}, \cdots t_{n}\right)^{\prime}$ for $n$ individuals are independent identically distributed (i.i.d.), and each corresponds to an exponential distribution with failure rate $\lambda$, where $\lambda>0$. Therefore, the p.d.f. is $f\left(t_{i} \mid \lambda\right)=\lambda \exp \left(-\lambda t_{i}\right)$. Correspondingly, the cumulative distribution function (c.d.f.) $F\left(t_{i} \mid \lambda\right)$ and the reliability function $R\left(t_{i} \mid \lambda\right)$ are $F\left(t_{i} \mid \lambda\right)=1-\exp \left(-\lambda t_{i}\right)=1-R\left(t_{i} \mid \lambda\right)$. Let $\boldsymbol{v}=\left(v_{1}, v_{2}, \cdots, v_{n}\right)^{\prime}$ indicate whether the lifetime is censored or not, and let the observed data set for current study be denoted as $D_{0}$, where $D_{0}=(n, t, v)$; following equation (1), the likelihood function related to $\lambda$ is given by

$$
L\left(\lambda \mid D_{0}\right)=\prod_{i=1}^{n}\left[\lambda \exp \left(-\lambda t_{i}\right)\right]^{v_{i}}\left[\exp \left(-\lambda t_{i}\right)\right]^{1-v_{i}} .
$$

Suppose $\mathbf{x}_{i}=\left(x_{1 i}, \cdots x_{p i}\right)^{\prime}$ denotes the $i^{\text {th }}$ individual of the $p \times 1$ vector of covariates; $\mathbf{X}$ is the $n \times p$ vector of covariates studied in reliability analysis, where $p$ denotes the quantity of the considered covariates. Suppose $\boldsymbol{\beta}$ is a $p \times 1$ vector of regression coefficients, representing the degree of the covariates' influence. Let $\lambda_{i}=\exp \left(\mathbf{x}_{i}^{\prime} \boldsymbol{\beta}\right)$, and the data set for the current study be denoted by $D$, where $D=(n, \mathbf{t}, \mathbf{X}, \boldsymbol{v})$. Following equation (2), the joint likelihood function for the exponential regression model is given by

$$
\begin{aligned}
L(\boldsymbol{\beta} \mid D) & =\prod_{i=1}^{n}\left[\exp \left(\mathbf{x}_{\mathbf{i}}^{\prime} \boldsymbol{\beta}\right) \exp \left(-\exp \left(\mathbf{x}_{\mathbf{i}}^{\prime} \boldsymbol{\beta}\right) t_{i}\right)\right]^{v_{i}}\left[\exp \left(-\exp \left(\mathbf{x}_{\mathbf{i}}^{\prime} \boldsymbol{\beta}\right) t_{i}\right)\right]^{1-v_{i}} \\
& =\exp \left[\sum_{i=1}^{n} v_{i} \mathbf{x}_{\mathbf{i}}^{\prime} \boldsymbol{\beta}\right] \exp \left[-\sum_{i=1}^{n} \exp \left(\mathbf{x}_{\mathbf{i}}^{\prime} \boldsymbol{\beta}\right) t_{i}\right] .
\end{aligned}
$$

The prior distributions should be realistic and computationally feasible. There are two common choices for $\boldsymbol{\beta}$ 's prior distribution. One is uniform improper prior distribution, for example, $\pi(\boldsymbol{\beta}) \propto 1$; the other is normal distribution. As proved by lbrahim et al. ${ }^{16}$, the latter is a log-concave prior distribution and is convenient for the computation of the posterior distribution. In this paper, we assume a multinormal prior distribution $\boldsymbol{\beta} \sim N_{p}\left(\boldsymbol{\mu}_{\mathbf{0}}, \boldsymbol{\Sigma}_{\mathbf{0}}\right)$, with mean $\boldsymbol{\mu}_{\mathbf{0}}$ and covariance matrix $\boldsymbol{\Sigma}_{\mathbf{0}}$. Let $\pi(\cdot)$ denote the prior or posterior distributions for the parameters; then, the joint posterior distribution $\pi(\boldsymbol{\beta} \mid D)$ can be written as

$$
\begin{aligned}
\pi(\boldsymbol{\beta} \mid D) \propto L(\boldsymbol{\beta} \mid D) \times \pi\left(\boldsymbol{\beta} \mid \boldsymbol{\mu}_{\mathbf{0}}, \boldsymbol{\Sigma}_{\mathbf{0}}\right) & \propto \exp \left[\sum_{i=1}^{n} v_{i} \mathbf{x}_{\mathbf{i}}^{\prime} \boldsymbol{\beta}\right] \exp \left[-\sum_{i=1}^{n} \exp \left(\mathbf{x}_{\mathbf{i}}^{\prime} \boldsymbol{\beta}\right) t_{i}\right] \times \exp \left[-\frac{1}{2}\left(\boldsymbol{\beta}-\boldsymbol{\mu}_{\mathbf{0}}\right)^{\prime} \boldsymbol{\Sigma}_{\mathbf{0}}^{-1}\left(\boldsymbol{\beta}-\boldsymbol{\mu}_{\mathbf{0}}\right)\right] \\
& =\exp \left[\sum_{i=1}^{n} v_{i} \mathbf{x}_{\mathbf{i}}^{\prime} \boldsymbol{\beta}-\sum_{i=1}^{n} \exp \left(\mathbf{x}_{\mathbf{i}}^{\prime} \boldsymbol{\beta}\right) t_{i}-\frac{1}{2}\left(\boldsymbol{\beta}-\boldsymbol{\mu}_{\mathbf{0}}\right)^{\prime} \boldsymbol{\Sigma}_{\mathbf{0}}^{-1}\left(\boldsymbol{\beta}-\boldsymbol{\mu}_{\mathbf{0}}\right)\right]
\end{aligned}
$$

Obviously, it is not easy to get the exact integration results for $\pi(\boldsymbol{\beta} \mid D)$ due to its complexity. Therefore, we select the MCMC method with the Gibbs sampler, which has been widely applied to Bayesian statistics since the 1990s, to carry out the posterior inference. Let $(-j)$ denote some vector without the $j^{\text {th }}$ component. The $j^{\text {th }}$ full conditional distribution can be written as

$$
\pi\left(\beta_{j} \mid D, \boldsymbol{\beta}^{(-j)}\right) \propto L\left(\beta_{j}, \boldsymbol{\beta}^{(-j)} \mid D\right) \times \pi\left(\beta_{j}, \boldsymbol{\beta}^{(-j)} \mid \boldsymbol{\mu}_{\mathbf{0}}, \boldsymbol{\Sigma}_{\mathbf{0}}\right) .
$$

\subsection{Bayesian Weibull Regression Model}

Suppose the lifetimes $\mathbf{t}=\left(t_{1}, \cdots t_{n}\right)^{\prime}$ for $n$ individuals are i.i.d., and each corresponds to a two-parameter Weibull distribution $W(\alpha, \gamma)$, where $\alpha>0$ and $\gamma>0$. The p.d.f. is $f\left(t_{i} \mid \alpha, \gamma\right)=\alpha \gamma t_{i}^{\alpha-1} \exp \left(-\gamma t_{i}^{\alpha}\right)$ while the c.d.f. $F\left(t_{i} \mid \alpha, \gamma\right)$ and the reliability function $R\left(t_{i} \mid \alpha, \gamma\right)$ are $F\left(t_{i} \mid \alpha, \gamma\right)=1-\exp \left(-\gamma t_{i}^{\alpha}\right)=1-R\left(t_{i} \mid \alpha, \gamma\right)$. To facilitate the analysis, let $\xi=\ln (\gamma)$ (note: it also can be viewed as an extreme value distribution). Then, the reliability function becomes

$$
f\left(t_{i} \mid \alpha, \xi\right)=\alpha t_{i}^{\alpha-1} \exp \left(\xi-\exp (\xi) t_{i}^{\alpha}\right) .
$$

Similarly, we can get $F\left(t_{i} \mid \alpha, \xi\right)$ and $R\left(t_{i} \mid \alpha, \xi\right)$.

As discussed in section 3.2, the censoring indicators are denoted as $\boldsymbol{v}=\left(v_{1}, v_{2}, \cdots, v_{n}\right)^{\prime}$, and the observed data set is $D_{0}=(n, \mathbf{t}, \boldsymbol{v})$, following equation (2); therefore, the likelihood function for $\alpha$ and $\xi$ is

$$
L\left(\alpha, \xi \mid D_{0}\right)=\alpha \sum_{i=1}^{n} v_{i} \exp \left\{\sum_{i=1}^{n} v_{i} \xi+\sum_{i=1}^{n} v_{i}\left[(\alpha-1) \ln \left(t_{i}\right)-\exp (\xi) t_{i}^{\alpha}\right]\right\} .
$$

To construct the Weibull Regression Model, we introduce the covariates through $\xi$. Denoting $\xi_{i}=\mathbf{x}_{\mathbf{i}}^{\prime} \boldsymbol{\beta}$ and following other definitions in section 3.2 , the joint likelihood function for the Weibull regression model is given by 


$$
L(\alpha, \boldsymbol{\beta} \mid D)=\alpha \sum_{i=1}^{n} v_{i} \exp \left\{\sum_{i=1}^{n} v_{i}\left[\mathbf{x}_{\mathbf{i}}^{\prime} \boldsymbol{\beta}+v_{i}(\alpha-1) \ln \left(t_{i}\right)-\exp \left(\mathbf{x}_{\mathbf{i}}^{\prime} \boldsymbol{\beta}\right) t_{i}^{\alpha}\right]\right\} .
$$

In this paper, we take $\alpha$ and $\xi$ to be independent. Furthermore, we assume $\alpha$ to be a gamma distribution denoted by $G\left(a_{0}, b_{0}\right)$ as its prior distribution, written as $\pi\left(\alpha \mid a_{0}, b_{0}\right)$, which means

$$
\pi\left(\alpha \mid a_{0}, b_{0}\right) \propto \alpha^{a_{0}-1} \exp \left(-b_{0} \alpha\right)
$$

Assume $\boldsymbol{\beta}$ has a multinormal prior distribution $\pi\left(\boldsymbol{\beta} \mid \boldsymbol{\mu}_{\mathbf{0}}, \boldsymbol{\Sigma}_{\mathbf{0}}\right)$ with $p$ vector, denoted by $N_{p}\left(\boldsymbol{\mu}_{\mathbf{0}}, \boldsymbol{\Sigma}_{\mathbf{0}}\right)$. Therefore, the joint posterior distribution can be obtained as

$$
\begin{aligned}
\pi(\alpha, \boldsymbol{\beta} \mid D) & \propto L(\alpha, \boldsymbol{\beta} \mid D) \times \pi\left(\alpha \mid a_{0}, b_{0}\right) \times \pi\left(\boldsymbol{\beta} \mid \boldsymbol{\mu}_{\mathbf{0}}, \boldsymbol{\Sigma}_{\mathbf{0}}\right) \\
& \propto \alpha^{a_{0}-1+\sum_{i=1}^{n} v_{i}} \exp \left\{\sum_{i=1}^{n}\left[v_{i} \mathbf{x}_{\mathbf{i}}^{\prime} \boldsymbol{\beta}+v_{i}(\alpha-1) \ln \left(t_{i}\right)-\exp \left(\mathbf{x}_{\mathbf{i}}^{\prime} \boldsymbol{\beta}\right) t_{i}^{\alpha}\right]-b_{0} \alpha-\frac{1}{2}\left(\boldsymbol{\beta}-\boldsymbol{\mu}_{\mathbf{0}}\right)^{\prime} \boldsymbol{\Sigma}_{\mathbf{0}}^{-1}\left(\boldsymbol{\beta}-\boldsymbol{\mu}_{\mathbf{0}}\right)\right\}
\end{aligned}
$$

Then, the parameters' full conditional distribution with Gibbs sampling can be written as

$$
\begin{gathered}
\pi\left(\alpha_{j} \mid \alpha^{(-j)}, \boldsymbol{\beta}, D\right) \propto L(\alpha, \boldsymbol{\beta} \mid D) \times \alpha^{a_{0}-1} \exp \left(-b_{0} \alpha\right) \\
\pi\left(\beta_{j} \mid \alpha, \boldsymbol{\beta}^{(-j)}, D\right) \propto L(\alpha, \boldsymbol{\beta} \mid D) \times \exp \left[\frac{1}{2}\left(\boldsymbol{\beta}-\boldsymbol{\mu}_{\mathbf{0}}\right)^{\prime} \boldsymbol{\Sigma}_{\mathbf{0}}^{-1}\left(\boldsymbol{\beta}-\boldsymbol{\mu}_{\mathbf{0}}\right)\right] .
\end{gathered}
$$

\subsection{Bayesian Log-normal Regression Model}

Suppose the lifetimes $\mathbf{t}=\left(t_{1}, \cdots t_{n}\right)^{\prime}$ for $n$ individuals are i.i.d., and each $\ln (t)$ corresponds to a normal distribution $N\left(\mu, \sigma^{2}\right)$. We can get $t_{i}^{\prime} s$ log-normal distribution with parameters $\mu$ and $\sigma^{2}$, denoted by $\operatorname{LN}\left(\mu, \sigma^{2}\right)$. Then, the p.d.f. and reliability function are given by

$$
\begin{gathered}
f\left(t_{i} \mid \mu, \sigma^{2}\right)=\frac{1}{\sqrt{2 \pi} \sigma t_{i}} \exp \left\{-\frac{1}{2 \sigma^{2}}\left[\ln \left(t_{i}\right)-\mu\right]^{2}\right\} ; \\
R\left(t_{i} \mid \mu, \sigma^{2}\right)=1-\Phi\left[\frac{\ln \left(t_{i}\right)-\mu}{\sigma}\right] .
\end{gathered}
$$

The likelihood function related to $\mu$ and $\sigma$, considering the censoring indicators $\boldsymbol{v}=\left(v_{1}, v_{2}, \cdots, v_{n}\right)^{\prime}$ and the observed data $D_{0}=(n, \mathbf{t}, \boldsymbol{v})$, becomes

$$
L\left(\mu, \sigma \mid D_{0}\right)=\left(2 \pi \sigma^{2}\right)^{-\frac{1}{2} \sum_{i=1}^{n} v_{i}} \exp \left\{-\frac{1}{2 \sigma^{2}}\left[\ln \left(t_{i}\right)-\mu\right]^{2}\right\} \times \prod_{i=1}^{n} t_{i}^{-v_{i}}\left\{1-\Phi\left[\frac{\log \left(t_{i}\right)-\mu}{\sigma}\right]\right\}^{1-v_{i}} .
$$

To construct a log-normal regression model, the covariates through $\mu$ are introduced with $\mu_{i}=\mathbf{x}_{\mathbf{i}}^{\prime} \boldsymbol{\beta}$. Letting $\tau=1 / \sigma^{2}$, the joint likelihood function is given by

$$
L(\boldsymbol{\beta}, \tau \mid D)=\left(2 \pi \tau^{-1}\right)^{-\frac{1}{2}} \sum_{i=1}^{n} v_{i} \exp \left\{-\frac{\tau}{2} \sum_{i=1}^{n} v_{i}\left[\left(\ln \left(t_{i}\right)-\mathbf{x}_{\mathbf{i}}^{\prime} \boldsymbol{\beta}\right)\right]^{2}\right\} \times \prod_{i=1}^{n} t_{i}^{-v_{i}}\left\{1-\Phi\left[\frac{\ln \left(t_{i}\right)-\mathbf{x}_{\mathbf{i}}^{\prime} \boldsymbol{\beta}}{\tau^{-1 / 2}}\right]\right\}^{1-v_{i}} .
$$

As both $\mu$ and $\tau$ are assumed unknown, a typical choice for $\tau$ is a gamma prior distribution. In this paper, we suppose $\tau \sim G\left(a_{0} / 2, b_{0} / 2\right)$. Meanwhile, as $\mu_{i}=\mathbf{x}_{\mathbf{i}}^{\prime} \boldsymbol{\beta}$, we also suppose $\boldsymbol{\beta}$ has a multinormal prior distribution with $p$ vector, denoted by $N_{p}\left(\boldsymbol{\mu}_{\mathbf{0}}, \tau^{-1} \Sigma_{\mathbf{0}}\right)$. The joint posterior distribution for $\tau$ and $\boldsymbol{\beta}$ can be obtained as

$$
\begin{aligned}
\pi(\boldsymbol{\beta}, \tau \mid D) & \propto L(\boldsymbol{\beta}, \tau \mid D) \times \pi\left(\tau \mid a_{0} / 2, b_{0} / 2\right) \times \pi\left(\boldsymbol{\beta} \mid \boldsymbol{\mu}_{\mathbf{0}}, \tau^{-1} \boldsymbol{\Sigma}_{\mathbf{0}}\right) \\
& \propto \frac{a_{0}+\sum_{i=1}^{n} v_{i}}{2}-1 \exp \left\{-\frac{\tau}{2} \sum_{i=1}^{n} v_{i}\left[\ln \left(t_{i}\right)-\mathbf{x}_{\mathbf{i}}^{\prime} \boldsymbol{\beta}\right]^{2}+\left(\boldsymbol{\beta}-\boldsymbol{\mu}_{\mathbf{0}}\right)^{\prime} \boldsymbol{\Sigma}_{\mathbf{0}}^{-\mathbf{1}}\left(\boldsymbol{\beta}-\boldsymbol{\mu}_{\mathbf{0}}\right)+b_{0}\right\} . \\
& \times \prod_{i=1}^{n} t_{i}^{-v_{i}}\left\{1-\Phi\left[\frac{\ln \left(t_{i}\right)-\mathbf{x}_{\mathbf{i}}^{\prime} \boldsymbol{\beta}}{\tau^{-1 / 2}}\right]\right\}^{1-v_{i}}
\end{aligned}
$$

Therefore, the parameters' full conditional distribution with Gibbs sampling can be written as

$$
\begin{gathered}
\pi\left(\tau_{j} \mid \tau^{(-j)}, \boldsymbol{\beta}, D\right) \propto L(\boldsymbol{\beta}, \tau \mid D) \times \tau^{\left(a_{0} / 2\right)-1} \exp \left(-b_{0} \tau / 2\right) \\
\pi\left(\beta_{j} \mid \boldsymbol{\beta}^{(-j)}, \tau, D\right) \propto L(\boldsymbol{\beta}, \tau \mid D) \times \exp \left\{\frac{1}{2}\left(\boldsymbol{\beta}-\boldsymbol{\mu}_{\mathbf{0}}\right)^{\prime}\left(\tau \boldsymbol{\Sigma}_{0}\right)^{-1}\left(\boldsymbol{\beta}-\boldsymbol{\mu}_{\mathbf{0}}\right)\right\} .
\end{gathered}
$$




\section{Case study}

In this section, we present a case study to illustrate our models for locomotive wheels' degradation analysis. The adopted data have been collected from a Swedish railway company's cargo locomotives. The studied locomotive is relatively new compared to others owned by the same company. The degradation data are reported from November 2010 to January 2012. There are 46 records $(n=46)$; we obtained the locomotive wheels' 'lifetime' data in the manner described in section 2.2 and shown in Figure 3. In this study, we define $\mathrm{H}_{2}=20 \mathrm{~mm}$; therefore, 12 records are denoted as censored data.

For each reported datum, a wheel's installation position is documented, and as mentioned above, positioning is used in this study as a covariate. As discussed in section 3, the wheel's position (bogie, axel and side) or covariate $\mathbf{X}$ is denoted by $x_{1}$ (bogie $\mathbf{l}$ : $x_{1}=1$, bogie II: $x_{1}=2$ ), $x_{2}$ (axel 1: $x_{2}=1$, axel 2: $x_{2}=2$, axel $3, x_{2}=3$ ) and $x_{3}$ (right: $x_{3}=1$, left: $x_{3}=2$ ). Correspondingly, the covariates' coefficients are represented by $\beta_{1}, \beta_{2}$, and $\beta_{3}$. In addition, $\beta_{0}$ is defined as a constant intercept. Other statistics on the wheel's position and the data structure appear in Table I.

The calculations are implemented with the software WinBUGS, version 1.4 (Spiegelhalter et al. ${ }^{22}$ ). A three-chain is constructed for each MCMC simulation. A burn-in of 10,001 samples is used, with an additional 10,000 Gibbs samples for each Markov chain. Vague prior distributions are adopted here as the following:

- In Bayesian Exponential Regression model:

$$
\beta_{0} \sim N(0,0.0001), \beta_{1}^{\sim} N(0,0.0001), \beta_{2} \sim N(0,0.0001), \beta_{3} \sim N(0,0.0001) .
$$

- In Bayesian Weibull Regression Model:

$$
\alpha^{\sim} G(0.2,0.2), \beta_{0} \sim N(0,0.0001), \beta_{1} \sim N(0,0.0001), \beta_{2} \sim N(0,0.0001), \beta_{3} \sim N(0,0.0001) .
$$

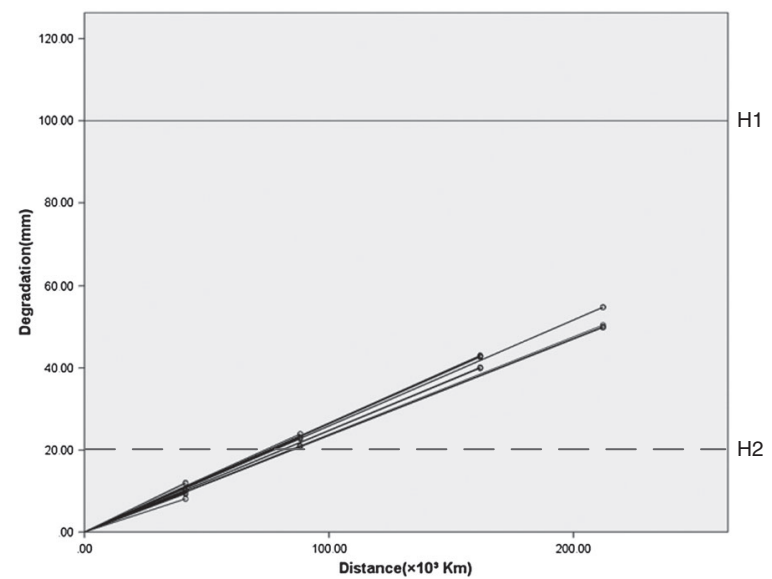

\begin{tabular}{|c|c|c|c|c|c|c|}
\hline & \multicolumn{2}{|c|}{$x_{1}$ : Bogie } & \multicolumn{2}{|c|}{$x_{2}:$ Axel } & \multicolumn{2}{|c|}{$x_{3}:$ Side } \\
\hline & Position & Quantities & Position & Quantities & Position & Quantities \\
\hline \multirow[t]{12}{*}{$n=46$} & I $(1)^{a}$ & 24 & $1(1)$ & 8 & Right (1) & 4 \\
\hline & & & & & Left (2) & 4 \\
\hline & & & $2(2)$ & 8 & Right (1) & 4 \\
\hline & & & & & Left (2) & 4 \\
\hline & & & $3(3)$ & 8 & Right (1) & 4 \\
\hline & & & & & Left (2) & 4 \\
\hline & II (2) & 22 & $1(1)$ & 8 & Right (1) & 4 \\
\hline & & & & & Left (2) & 4 \\
\hline & & & $2(2)$ & 8 & Right (1) & 4 \\
\hline & & & & & Left (2) & 4 \\
\hline & & & $3(3)$ & 6 & Right(1) & 3 \\
\hline & & & & & Left (2) & 3 \\
\hline
\end{tabular}

Figure 3. Plot of the wheel degradation data

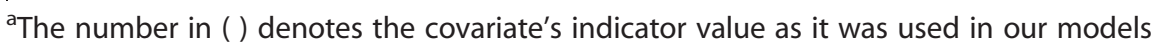


- In Bayesian Log-normal Regression Model:

$$
\tau^{\sim} G(1,0.01), \beta_{0}^{\sim} N(0,0.0001), \beta_{1}^{\sim} N(0,0.0001), \beta_{2}^{\sim} N(0,0.0001) \beta_{3}^{\sim} N(0,0.0001) .
$$

Following the convergence diagnostics (i.e. checking dynamic traces in Markov chains, time series and Gelman-Rubin-Statistics, and comparing the MC error with standard deviation (SD)), we consider the following posterior distribution summaries (shown in Tables II, III and IV), for our models (Bayesian Exponential Regression Model, Bayesian Weibull Regression Model and Bayesian Lognormal Regression Model), including the parameters' posterior distribution mean, SD, Monte Carlo error and 95\% highest posterior distribution density (HPD) interval.

Accordingly, the locomotive wheels' reliability functions can be written as:

- Bayesian Exponential Regression Model:

$$
R\left(t_{i} \mid \mathbf{X}\right)=\exp \left[-\exp \left(-5.862-0.072 x_{1}-0.032 x_{2}-0.012 x_{3}\right) \times t_{i}\right]
$$

- Bayesian Weibull Regression Model:

$$
R\left(t_{i} \mid \mathbf{X}\right)=\exp \left[-\exp \left(-60.47-0.078 x_{1}-0.146 x_{2}-0.050 x_{3}\right) \times t_{i}^{10.08}\right]
$$

- Bayesian Log-normal Regression Model:

$$
R\left(t_{i} \mid \mathbf{X}\right)=1-\Phi\left[\frac{\ln \left(t_{i}\right)-\left(5.864+0.067 x_{1}+0.02 x_{2}+0.001 x_{3}\right)}{(187.5)^{-1 / 2}}\right]
$$

\begin{tabular}{|c|c|c|c|c|}
\hline Parameter & Mean & SD & MC error & 95\% HPD interval \\
\hline$\alpha$ & 10.08 & 0.9674 & 0.05559 & $(8.234,11.76)$ \\
\hline$\beta_{0}$ & -60.47 & 5.977 & 0.3434 & $(-71.01,-49.16)$ \\
\hline$\beta_{1}$ & -0.07775 & 0.306 & 0.008339 & $(-0.6845,0.5156)$ \\
\hline$\beta_{2}$ & -0.146 & 0.2231 & 0.005801 & $(-0.5878,0.2856)$ \\
\hline$\beta_{3}$ & -0.05026 & 0.2982 & 0.007143 & $(-0.6356,0.5324)$ \\
\hline
\end{tabular}

\begin{tabular}{|c|c|c|c|c|}
\hline Parameter & Mean & SD & $\mathrm{MC}$ error & 95\% HPD interval \\
\hline$\beta_{0}$ & 5.864 & 0.05341 & 0.001622 & $(5.76,5.97)$ \\
\hline$\beta_{1}$ & 0.06733 & 0.02174 & $5.042 \mathrm{E}-4$ & $(0.02492,0.1103)$ \\
\hline$\beta_{2}$ & 0.02077 & 0.01373 & $2.765 \mathrm{E}-4$ & $(-0.006291,0.04781)$ \\
\hline$\beta_{3}$ & 0.001102 & 0.02175 & $5.007 \mathrm{E}-4$ & $(-0.0412,0.04444)$ \\
\hline$\tau$ & 187.5 & 39.84 & 0.3067 & $(118.3,273.5)$ \\
\hline
\end{tabular}

Obviously, other reliability characteristics of lifetime distribution, including MTTF, can also be determined.

\begin{tabular}{|lllll|}
\hline \multicolumn{4}{|l}{ Table II. Posterior distribution summaries for Exponential Regression Model } & \\
Parameter & Mean & SD & MC error & 95\% HPD interval \\
\hline$\beta_{0}$ & -5.862 & 0.7355 & 0.02299 & $(-7.366,-4.452)$ \\
$\beta_{1}$ & -0.07207 & 0.3005 & 0.007269 & $(-0.6672,0.5104)$ \\
$\beta_{2}$ & -0.03219 & 0.1858 & 0.003797 & $(-0.3889,0.3325)$ \\
$\beta_{3}$ & -0.0124 & 0.2973 & 0.00726 & $(-0.5954,0.5787)$ \\
\hline
\end{tabular}




\section{Discussion}

\subsection{Model comparison}

Traditional technologies for model comparison consider two main aspects: the model's measure of fit and its complexity. Usually, improving the model's complexity can simultaneously improve its fit. For instance, by considering more unknown parameters, the SD and MC error of the model's posterior could be reduced and the model's measure of fit could be improved. However, the complexity of the model will be increased simultaneously. Therefore, most model comparison studies focus on the balance between them. When comparing Bayesian models, both Bayesian Factor and Bayesian Information Criterion can be used. However, for complex Bayesian hierarchical models, it becomes more difficult. Spiegelhalter et al. ${ }^{23}$ have proposed DIC, which utilizes the model's deviance to evaluate its measure of fit, and the effective number of parameters to evaluate its complexity.

Define a Bayesian model's Bayesian deviance, denoted as $D(\theta)$, as:

$$
D(\theta)=-2 \log (p(D \mid \theta)) .
$$

Define the effective number of parameters, denoted as $p_{d}$, as:

$$
p_{d}=\bar{D}(\theta)-D(\bar{\theta})=-\int 2 \ln (p(D \mid \theta)) d \theta-(-2 \ln (p(D \mid \bar{\theta}))) .
$$

Then,

$$
D I C=D(\bar{\theta})+2 p_{d}=\bar{D}(\theta)+p_{d}
$$

We calculate the DIC values for the above three Bayesian parametric models separately, as shown in Table V.

Based on Celeux et al. ${ }^{24}$ and related discussions of their paper, we choose the model with the lowest DIC value. When DIC $<5$, the difference among models can be neglected. Our results show that the DIC for Log-normal Regression Model is the lowest (447.19), and following the arguments above, it is more suitable than the other two. In addition, we analyse other locomotives' wheels with the same model, which are running under similar situations. The results show that similar conclusions can be achieved. However, comparing the DIC values for Weibull Regression Model and for Exponential Regression Model, which is 477.05 and 652.93, separately, they indicate that the performance of Weibull Regression Model is close to the Log-normal Regression Model, which might also be a suitable choice under specified situations.

\subsection{Maintenance predictions}

Although there is a little difference among the different Bayesian parameter models, all results achieve consistent common conclusion: the installation positions influence the wheels' lifetimes. In addition, considering the character of the covariates' coefficients in our case study, we find the following: (i) the lifetime of the wheel installed in the second bogie is longer than that of the wheel installed in the first one; (ii) the wheel installed in the third axel has a longer lifetime than that installed in the second axel, and the wheel in the second axel has a longer lifetime than the one in the first axel; (iii) the right side wheel's lifetime is shorter than the left side. (Researchers from Norwegian National Rail Administration cited previously concur with this. Using condition monitoring methods on train wheels operating on the same route, they found that the wheel forces on the right and the left sides can be different, even for wheels in the same axel.). Possible causes include the influence of the topographical complexity and the position of the locomotive's centre of gravity.

The three Bayesian parametric regression models presented here are all effective according to Markov chain convergence and other diagnostic tools; see, for example, Spiegelhalter et al. ${ }^{23}$ who compare the computation process, including checking Markov chains' dynamic traces, time series and Gelman-Rubin-Statistics, and comparing the MC error with SD. However, we prefer Bayesian Log-normal Regression Model because of its DIC values. The prediction of the locomotive wheels MTTF, following Bayesian Lognormal Regression Model, appears in Table VI.

It should be pointed out that the 95\% HPD interval in Bayesian Log-normal Regression Model for $\beta_{2}$ and $\beta_{3}$ includes 0 (Table IV). This means that, although the positioning does have an influence, in some instances, the impact on the wheel's service lifetime is not

\begin{tabular}{|c|c|c|c|c|}
\hline Model & $\bar{D}(\theta)$ & $D(\bar{\theta})$ & $p_{d}$ & DIC \\
\hline Exponential Regression & 648.98 & 645.03 & 3.95 & 652.93 \\
\hline Weibull Regression & 472.22 & 467.39 & 4.83 & 477.05 \\
\hline Log-normal Regression & 442.03 & 436.87 & 5.16 & 447.19 \\
\hline
\end{tabular}
significantly strong. In our case, the bogies have more impact on service lifetime than axels or sides. Given this conclusion, we can deal with such covariates better in our future research. 


\begin{tabular}{|c|c|c|c|c|}
\hline Bogie & Axel & Side & $\mu_{i}$ & $\operatorname{MTTF}\left(\times 10^{3} \mathrm{~km}\right)$ \\
\hline \multirow[t]{6}{*}{ I $\left(x_{1}=1\right)$} & \multirow{2}{*}{$1\left(x_{2}=1\right)$} & Right $\left(x_{3}=1\right)$ & 5.9532 & 387.03 \\
\hline & & Left $\left(x_{3}=2\right)$ & 5.9543 & 387.46 \\
\hline & \multirow{2}{*}{$2\left(x_{2}=2\right)$} & Right $\left(x_{3}=1\right)$ & 5.9740 & 395.16 \\
\hline & & Left $\left(x_{3}=2\right)$ & 5.9751 & 395.60 \\
\hline & \multirow{2}{*}{$3\left(x_{2}=3\right)$} & Right $\left(x_{3}=1\right)$ & 5.9947 & 403.43 \\
\hline & & Left $\left(x_{3}=2\right)$ & 5.9958 & 403.87 \\
\hline \multirow[t]{6}{*}{ II $\left(x_{1}=2\right)$} & \multirow[t]{2}{*}{$1\left(x_{2}=1\right)$} & Right $\left(x_{3}=1\right)$ & 6.0205 & 413.97 \\
\hline & & Left $\left(x_{3}=2\right)$ & 6.0216 & 414.43 \\
\hline & \multirow[t]{2}{*}{$2\left(x_{2}=2\right)$} & Right $\left(x_{3}=1\right)$ & 6.0413 & 422.67 \\
\hline & & Left $\left(x_{3}=2\right)$ & 6.0424 & 423.14 \\
\hline & \multirow[t]{2}{*}{$3\left(x_{2}=3\right)$} & $\operatorname{Right}\left(x_{3}=1\right)$ & 6.0621 & 431.56 \\
\hline & & Left $\left(x_{3}=2\right)$ & 6.0632 & 432.03 \\
\hline
\end{tabular}
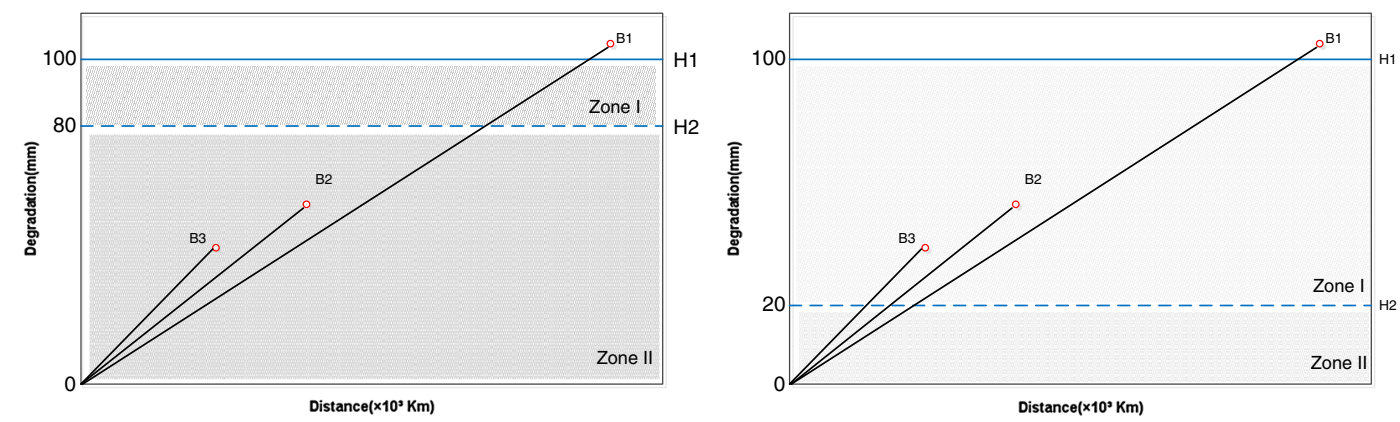

Figure 4. Maintenance inspection level with Zone I and Zone II

\subsection{Maintenance inspection level}

According to the assumptions in section 2.2, the maintenance inspection level $H_{2}$ (where $0 \leq H_{2} \leq H_{1}$ ) determines how many lifetime data are 'right censored'. Obviously, the higher the maintenance inspection level, the more data are considered 'right censored' and vice versa. For instance, in Figure 4, we show a higher maintenance inspection level $(80 \mathrm{~mm})$ and a lower one $(20 \mathrm{~mm})$. We denote the area between $H_{1}$ and $H_{2}$ as Zone I, and the area between $H_{2}$ and zero degradation level as Zone II. Therefore, based on the likelihood functions discussed in section 3, the MTTF statistics which are achieved from the higher $\mathrm{H}_{2}$ (the left picture in Figure 4, where $\mathrm{H}_{2}=80 \mathrm{~mm}$ ) will be higher than those obtained from the lower $\mathrm{H}_{2}$ (the right picture, where $\mathrm{H}_{2}=20 \mathrm{~mm}$ ), because fewer degradation data are considered right censored. In other words, the results achieved from the former are more 'optimistic', and the results obtained from the latter are more 'pessimistic'. An extreme condition is to suppose $\mathrm{H}_{2}=0 \mathrm{~mm}$.

For this reason, we can get an interval prediction between 'optimistic' and 'pessimistic' with different maintenance inspection levels, which actually reflect the different risk confidence levels. This will be studied in another research paper.

\section{Conclusions}

This paper proposes three parametric Bayesian models for locomotive wheels' reliability analysis using degradation data: Bayesian Exponential Regression Model, Bayesian Weibull Regression Model (as discussed in section 3.3.1, it can easily be transferred to an Extreme-Value Regression Model) and Log-normal Regression Model. The Bayesian survival models can deal with small and incomplete data sets and simultaneously consider the influence of several covariates.

The case study's results suggest that the wheels' lifetimes differ according to where they are installed on the locomotive. The wheel installed in the second bogie has a longer lifetime than the one installed in the first bogie; the one installed in the 'back' axel has a longer lifetime than the 'front' one; the right and left side wheels also differ. The differences between the latter two could be influenced by many aspects, for instance, the locomotives' heterogeneities, the real running situation (e.g. topography, temperature, moisture, applied loading, train speed, etc.) and the locomotive's centre of gravity. However, the bogies have the strongest influence on wheel lifetime. We can determine the wheel's MTTF using the prediction results obtained from equation (20) (22); this, in turn, allows us to evaluate and optimise the wheel's replacement and maintenance strategies (including the re-profiling interval, inspection interval, lubrication interval and so on). In addition, by defining different maintenance inspection levels, we can obtain an interval prediction between 'optimistic' and 'pessimistic' with different risk confidence levels. 
Finally, the approach discussed in this paper can be applied to cargo train wheels or to other technical problems (e.g. other industries, other components).

The study suggests the following additional research:

- The assumed linear degradation path is a simple one. For more complex path models, more degradation paths should be studied.

- The covariates considered in this paper are limited to locomotive wheels' installed positions; more covariates must be considered.

- We have chosen vague prior distributions for the case study. Other prior distributions, including both informative and non-information prior distributions, should be studied.

\section{Acknowledgements}

The authors would like to thank Luleå Railway Research Centre (Järnvägstekniskt Centrum, Sweden) and Swedish Transport Administration (Trafikverket) for initiating the research study and providing financial support. Also, the authors would like to thank Thomas Nordmark, Ove Salmonsson and Hans-Erik Fredriksson at LKAB for support and discussions about the locomotive wheels. And we would like to thank the editor and anonymous referees for their constructive comments.

\section{References}

1. Johansson A, Andersson C. Out-of-round Railway Wheels- a Study of Wheel Polygonalization through Simulation of Three-dimensional Wheel-Rail Interaction and Wear. Journal of Vehicle System Dynamics 2005; 43(8):539-559. DOI: 10.1080/00423110500184649

2. Braghin F, et al. A Mathematical Model to Predict Railway Wheel Profile Evolution Due to Wear. Journal of Wear 2006; 261:1253-1264. DOI: 10.1016/ j.wear.2006.03.025

3. Tassini N, et al. A Numerical Model of Twin Disc Test Arrangement for the Evaluation of Railway Wheel Wear Prediction Methods. Journal of Wear 2010; 268:660-667. DOI: 10.1016/j.wear.2009.11.003

4. Bernasconi A, et al. An Integrated Approach to Rolling Contact Sub-surface Fatigue assessment of Railway Wheels. Journal of Wear 2005; 258:973980. DOI: 10.1016/j.wear.2004.03.044

5. Liu YM, et al. Multiaxial Fatigue Reliability Analysis of Railroad Wheels. Journal of Reliability Engineering and System Safety 2008; 93:456-467. DOI: 10.1016/j.ress.2006.12.021

6. Clayton P. Tribological Aspects of Wheel-Rail Contact: A Review of Recent Experimental Research. Journal of Wear 1996; 191:170-183. DOI: 10.1016/ 0043-1648(95)06651-9

7. Yang C, Letourneau S. Learning to Predict Train Wheel Failures. Conference Proceedings. The 11th ACM SIGKDD International Conference on Knowledge Discovery and Data Mining (KDD 2005). Chicago, Illinois, USA.

8. Pombo J, Ambrosio J, Pereira M. A Railway Wheel Wear Prediction Tool based on A Multibody Software. Journal of Theoretical and Applied Mechanics 2010; 48(3):751-770.

9. Skarlatos D, Karakasis K, Trochidis A. Railway Wheel Fault Diagnosis Using A Fuzzy-logic Method. Journal of Applied Acoustics 2004; 65:951-966. DOI: 10.1016/j.apacoust.2004.04.003

10. Donato P, et al. Design and Signal Processing of A Magnetic Sensor Array for Train Wheel Detection. Journal of Senors and Actuators A 2006; 132:516-525. DOI: 10.1016/j.sna.2006.02.043

11. Stratman B, Liu Y, Mahadevan S. Structural Health Monitoring of Railroad Wheels Using Wheel Impact Load Detectors. Journal of Failure Analysis and Prevention 2007; 7(3):218-225. DOI: 10.1007/s11668-007-9043-3

12. Palo $\mathrm{M}$, et al. Rolling stock condition monitoring using wheel/rail forces. Journal of Insight - Non-Destructive Testing and Condition Monitoring 2012; 54(8):451-455. DOI: 10.1784/insi.2012.54.8.451

13. Palo M. Condition Monitoring of Railway Vehicles: A Study on Wheel Condition for Heavy Haul Rolling Stock. Licentiate Thesis. Luleå University of Technology, Sweden, 2012; 37.

14. Freitas MA, et al. Using Degradation Data to Assess Reliability: A Case Study on Train Wheel Degradation. Journal of Quality and Reliability Engineering International 2009; 25:607-629. DOI: 10.1002/qre.995

15. Freitas MA, et al. Reliability assessment using degradation models: Bayesian and classical approaches. Pesquisa Operacional 2010; 30(1):195-219.

16. Ibrahim JG, Chen MH, Sinha D. Bayesian Survival Analysis. Springer, New York - Berlin - Heidelberg: 2001.

17. Congdon P. Applied Bayesian Modelling. John Wiley and Sons: England, 2003.

18. Congdon P. Bayesian Statistical Modelling. John Wiley and Sons: England, 2001.

19. Lin J. Two-Stage Failure Model for Bayesian Change Point Analysis. Journal of IEEE Transactions on Reliability 2008; 57(2):388-393. DOI: 10.1109/ TR.2008.923484

20. Klein JP, Moeschberger ML. Survival Analysis: Techniques for Censored and Truncated Data. Springer-Verlag Inc.: New York, $1997 ; 55$.

21. Lawless JF, Statistical Models and Methods for Lifetime Data. John Wiley \& Sons, Inc., 1982; 31.

22. Spiegelhalter D, et al. WinBUGS User Manual (Version 1.4). January, 2003. http:// www.mrc-bsu.cam.ac.uk/bugs [11 September 2012]

23. Spiegelhalter DJ, et al. Bayesian measures of model complexity and fit. Journal of Royal Statist. Society Series B 2002; 64(3):583-639. DOI: 10.1111/ $1467-9868.00353$

24. Celeux G, et al. Deviance Information Criteria for Missing Data Models. Journal of Bayesian Analysis 2006; 1(4):651-674

\section{Authors' biographies}

Dr. Jing Lin is currently a researcher in the Division of Operation and Maintenance Engineering, at Luleå University of Technology, Sweden. She obtained her PhD degree in Management from Nanjing University of Science and Technology (NJUST), China, in April 2008, and she received the bachelor degree in Management from (NJUST) in 2003. After college, she worked 3 years for SKF Co., 
Ltd as an Asset Management Consultant. Dr. Lin's research interests primarily lie in asset management and reliability. She has published 41 peer-reviewed Journal and Conference papers and one monograph in related topics.

Matthias Asplund is a PhD student in the Division of Operation and Maintenance Engineering, at Luleå University of Technology, Sweden, since 2011. His research area is RAMS (Reliablility, Availability, Maintainability and Safty) with railway topics in focus. He has 12 years working experience from product development, lean production, maintenance and railway engineering. He got his Master degree in Mechanical Engineering with focus on Applied Mechanics from Luleå Technical University. His last work before studies to PhD was Track Engineering for the Swedish Transport Administration.

Dr. Aditya Parida is an Associate Professor in the Division of Operation and Maintenance Engineering, at Luleå University of Technology, Sweden. He obtained his PhD in Operation and Maintenance Engineering. His area of research is Asset Management, Maintenance Performance Measurement and model, RCM and eMaintenance. Besides teaching, he is actively involved in research and projects. He is the author of 70 peer-reviewed Journal and Conference papers, besides three book chapters, and guest editor of four special issues of International Journals. 